\title{
Microhotplates for Low Power, and Ultra Dense Gaseous Sensor Arrays Using Recessed Silica Aerogel for Heat Insulation
}

\author{
Mohammad Seyed Jalali ${ }^{1}$, Sanjay Kumar ${ }^{2}$, Mohammad Madani ${ }^{2}$ and Nian-Feng Tzeng ${ }^{1}$ \\ ${ }^{1}$ The Center for Advanced Computer Studies, \\ University of Louisiana at Lafayette, Lafayette, LA, USA \\ Email: \{sxs5330, tzeng@ cacs.louisiana.edu\} \\ ${ }^{2}$ Microelectonics Research Lab at the Department of Electrical and Computer Engineering, \\ University of Louisiana at Lafayette, Lafayette, LA, USA \\ Email: \{sxl7123, madani@louisiana.edu\}
}

\begin{abstract}
In the operation of air pitted gaseous sensor the microhotplate $(\mu \mathrm{HP})$ consumes almost all the power used by the sensor. The required area to micromachine the air pit for the $\mu \mathrm{HP}$ of a single sensor is several times more than the actual area required by the sensor itself. The feasibility of implementing low power and ultra dense gaseous sensor array is investigated by developing a new $\mu \mathrm{HP}$ structure using recessed silica aerogel. In comparison with the conventional $\mu \mathrm{HP}$ structure, the recessed aerogel not only has decreased the utilized area of the chip almost ten folds $\left(181 \times 181{\mu m^{2}}^{2}\right.$ vs. $\left.573 \times 573 \mu^{2}\right)$ to maintain a temperature of $360^{\circ} \mathrm{C}$ but also has decreased the power consumed by each $\mu \mathrm{HP}$ more than two folds ( $1 \mathrm{~mW}$ vs. 2.1 $m W)$. As the number of sensors increases in a sensor array, the saved area of the chip increases quadratic by using the new structure. Moreover, the power consumed by the new designed structure reduces drastically.

Index Terms-Micromachining, low energy, gaseous sensor arrays, metal oxides, microhotplate, silica aerogel, recessed aerogel, step coverage.
\end{abstract}

\section{INTRODUCTION}

Silica aerogel has gained attention both in research and industrial communities due to its unique properties of ultralow thermal conductivity, high thermal stability, high specific surface area, etc. [1], [2]. Recently there have been several studies on aerogel applications in the various fields including but not limited to capacitive deionization of water [3]aero capacitor due to high surface area of aerogel [4], [5], and sound absorption in ultrasonic devices [6], [7]. More recently, aerogels have been successfully synthesized in different forms of microspheres [8], thin films [9], and flexible sheets [10], [11] among which thin films have received more interest in applications such as ideal dielectrics for ultrafast integrated circuits [12] and heat insulator in gaseous sensors [13] due to the low thermal conductivity of aerogel. Combining it with microelectromechanical systems (MEMS) expands the applications of aerogel even more in devices requiring a steady high temperature.

The Metal Oxide (MOX) gas sensors should operate at temperature range of $200{ }^{\circ} \mathrm{C}$ to $500{ }^{\circ} \mathrm{C}$ [13] for maximum sensitivity. The sensing material is deposited on a plate called microhotplate $(\mu \mathrm{HP})$. The high operating temperature of metal oxide (MOX) gas sensor demands an efficient design to consume low power in order to raise the temperature of $\mu \mathrm{HP}$ to desired level as quickly as possible. Various designs and materials have been considered for microhotplate fabrication [14]-[16]. Specifically, a micro hotplate fabricated with an active surface area of $50 \times 60 \mu^{2}$ was reported to consume $30 \mathrm{~mW}$ power when operating at $350{ }^{\circ} \mathrm{C}$ [16]. In another article, a circular active surface area of $80 \mu \mathrm{m}^{2}$ in diameter was developed to operate at higher temperature of $400{ }^{\circ} \mathrm{C}$ consuming some $8.9 \mathrm{~mW}$ only [14], [15]. The latter achieves significant improvement in power efficiency by increasing its pit height to $400 \mu \mathrm{m}$ based on front side bulk micromachining [15]. However, this power efficiency is at a significant expense of chip area taken by each sensor. By simple calculation one can quickly realize that minimum chip area needed to micromachine such a deep pit would be in order of $566 \times 566$ $\mu m^{2}$, which is 64 times the size of microhotplate itself. As a result, microsensor arrays obtained using such microhotplate design will undoubtedly suffer from a very low sensor density. Briand et al. [17] have reported to make microhotplate gas sensor on a polyimide layer (as insulating layer) to avoid tedious front micromachining involved in processing air pit microhotplate gas sensors. However, they end up with a hotplate size of $1.5 \mathrm{~mm}$ wide and power consumption of 66 $m W$ to reach the temperature of $325^{\circ} \mathrm{C}$. Therefore, we are in urgent need of area efficient gaseous sensor design with low power consumption to present a cost-effective manufacturing of sensor arrays on the wafer.

To overcome the limitation of low density sensor arrays and high power consumption to reach high temperature on microhotplate, we proposed a novel approach of using aerogel as heat insulator rather than using air. The conventional method to insulate the microhotplate from the silicon substrate is to micromachine the $\mathrm{Si}$ substrate to form an air pit. Wet micromachining is a post processing step, where over etching may occur while masking the sensor and its circuitry due to improper etching time control or pinholes in the masking mate- 
rial. This can reduce the yield and compromise the mechanical stability of the $\mu \mathrm{HP}$. During the fabrication process of $\mu \mathrm{HP}$, Laconte et al. [18] reported broken membranes due to backside micromachining with Tetra Methyl Ammonium Hydroxide (TMAH) etchant, which also damaged aluminum interconnections when masking layers failed. Furthermore, many of those surviving membranes were broken during subsequent deposition of the sensing layer material, photolithography processes, and selective wet etching. Dicing is another cause of the yield loss, since conventional dicing utilizes high water pressure to remove debris from the chip surface [19].

We have previously reported [13] that using relatively thick (40-100 $\mu \mathrm{m}$ ) aerogel material instead of air as heat insulator yields the following advantages: (1) ultra low power consumption, (2) area-efficient design to support high sensor density, (3) excellent temperature uniformity across the microhotplate surface, (4) low manufacturing costs (due to high yields), (5) high mechanical stability, and (6) fast fabrication. However, processing a thick layer of aerogel of $5 \mu \mathrm{m}$ or more is extremely difficult using multilayer processing since the spin coating of aerogel is limited to 0.6 to $3 \mu \mathrm{m}$ per layer [20]-[22]. In view of the fact that a relatively thick layer of aerogel is required for ultra low power MOX sensors, the step coverage problem for the metal interconnection lines between the sensor array and the CMOS chip circuitry will pose significant yield problem. However, we have demonstrated in this paper that the recessing of the thick aerogel in a selected area of the chip not only resolves the step coverage problem but also avoids tedious and difficult multilayer processing of thick aerogel film. Furthermore, the recessed aerogel processing will not adversely lower the yield caused by post processing of the sensor array.

\section{AIR PIT DESIGN AND CHIP AREA CONSIDERATIONS}

The whole structure of the air pit is made of 3 layers as depicted in Fig.1(a). At the bottom there is a $p$-type silicon substrate in which an air pit is created to provide thermal insulation. The first layer is a dense $2 \mu \mathrm{m}$ thick thermally grown $\mathrm{SiO}_{2}$ or $\mathrm{Si}_{3} \mathrm{~N}_{4}$ serving as micromachining mask shaped into four suspended bridges for mechanical support of the sensor shown in Fig.1(a). But in case of aerogel, this layer is a complete layer of $\mathrm{SiO}_{2}$ on top of the aerogel and the thickness can be as low as $0.2 \mu \mathrm{m}$ as illustrated in Fig.1(a). The second layer is the $\mathrm{NiCr}(\mathrm{Ni80} / \mathrm{Cr} 20)$ on top of $\mathrm{SiO}_{2}$ which is also $0.2 \mu \mathrm{m}$. This layer can be highly doped polysilicon for CMOS process compatibility. Finally, the third layer is an $\mathrm{SiO}_{2}$ layer of $0.6 \mu \mathrm{m}$ thickness to provide electrical insulation of heater from the micro hotplate. It also yields better temperature uniformity across the microhotplate since $\mathrm{SiO}_{2}$ is relatively a good heat conductive material. As reported in our previous study for the non-recessed spin coated thin aerogel, micromachining of the silicon is completely eliminated. Although the thicker aerogel reduces the power drastically, high step coverage decreases the yield severely. In this work we have investigated the recessed aerogel with micromachining a large area of silicon for sensor arrays prior to the fabrication of the sensor. Then we fill the anisotropically etched cavity with aerogel.
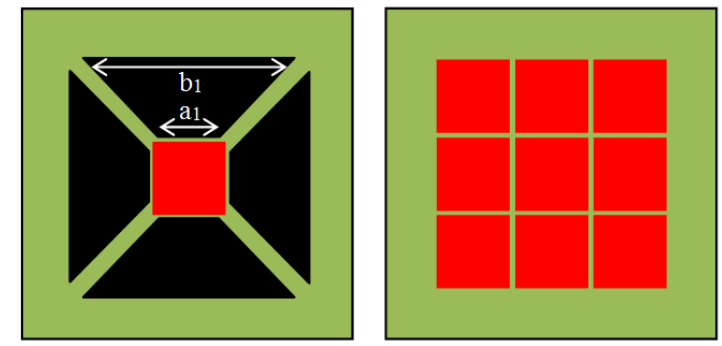

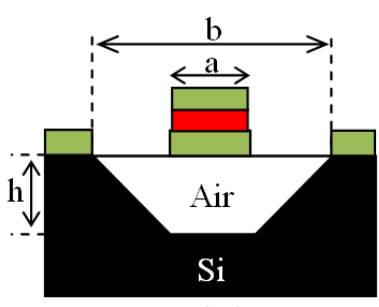

(a) Airpit

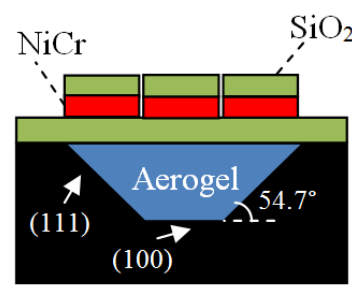

(b) Recessed Aerogel
Fig. 1. Design Schematic of (a) single $\mu \mathrm{HP}$ on airpit and (b) array of $\mu \mathrm{HP}$ on recessed aerogel.

Anisotropic etching is used to form the cavity underneath the hotplate of the gas sensor. The etching is called anisotropic since the etching rate is high in the (100) direction and low in the (111) direction. Etch rate in the two direction can be different as 300 to 1 [23]. In the silicon crystal lattice structure, the (111) planes are oriented at $54.7^{\circ}$ relative to the (100) plane (Fig.1(b)). A square mask opening on the surface of the wafer will yield an etched feature in the shape of inverted pyramid at the depth determined by the intersection of (111) plane. To suspend the microhotplate in the air a mask is made with four trapezoids (with dimensions: $a_{1}=$ short base, $b_{1}=$ long base, and $h_{T}=$ altitude) placed close together from their short bases $a_{1}$ to form an area of square of $a \times a$ where $a=$ $a_{1}+2 \Delta w$ for the hotplate (Fig.1(a)). The area considered for the hotplate is $181 \times 181 \mu \mathrm{m}^{2}(a=181)$. The four long bases of the trapezoids $b_{1}$ will form the mask opening area of $b \times b$ where $b=b_{1}+2 \Delta w$. The four straps that hold the micro hotplate suspended in the air after micromachining have the width $w=\sqrt{2} \Delta w$ and the length of straps $l=\frac{\sqrt{2}(b-a)}{2}$.

The thermo-electrical analysis of our designed structures is performed by utilizing a MEMS simulation software, IntelliSuite. The software is equipped with the following modules: IntelliMask to design the mask; 3D Builder to create the meshed solid blocks and differentiate their entities on different layers; TEM (ThermoElectroMechanical) to assign the properties of each entity, load the initial conditions, and simulate the temperature gain by applying voltage to one end of the heater, keeping the other end at zero potential; the IntelliEtch will figure out the final shape of micromachined structure by using an etchant like $\mathrm{KOH}$ buffer. The temperature at the bottom of the silicon substrate is set to room temperature of $27^{\circ} \mathrm{C}$ to resemble the reference temperature. Input power can then be calculated by knowing the applied voltage and reading the 
current density passed through the heater.

\section{RESUlTS AND Discussion}

To etch out the silicon from underneath of an $a \times a$ hotplate $h$ deep, the required square size opening is:

$$
b=a+\frac{2 h}{\tan \left(54.7^{\circ}\right)} .
$$

The etchant will etch the silicon through the opening area of the trapezoid to make the air pit as shown in Fig.1(a). On the other hand, in order to make the pit filled with aerogel there is no need of a trapezoid mask, but a simple square mask would create the pit as illustrated in Fig.1(b). According to (1) the area of the mask opening or the total area used increases as a square function of the height of the pit. Hence, one can calculate the percentage of saved area as:

$$
\frac{b^{2}-a^{2}}{a^{2}} \times 100,
$$

which implies that the micromachined air pit for each individual sensor in a sensor array uses much more area of the chip than using aerogel on the wafer for the same array. Fig.2 demonstrates the percentage of the saved area for different depths of the micromachined pit. For instance, by having a depth of only $160 \mu \mathrm{m}$ pit filled with aerogel, we can significantly save four times less area than that of air pit. In another word, for every sensor processed with air pit we can have 5 sensors using aerogel. As the height of the aerogel increases more space would be saved by a parabolic factor. Hence, a denser sensor array can be fabricated quite easily by using aerogel as compared to micromachined air pit.

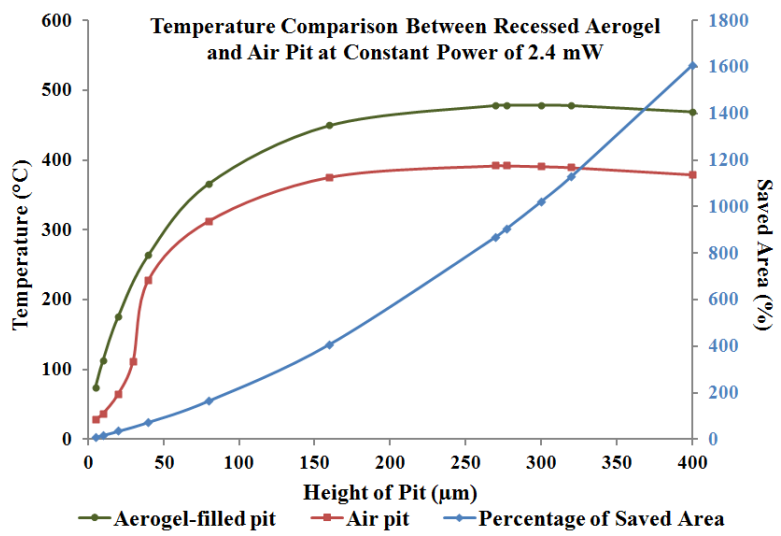

Fig. 2. Temperature and saved area for different heights of pit.

Besides, in order to build the air pit we are limited by the trapezoid openings in micromachining. For instant, up to a certain height $h$, a column of silicon will remain unetched as demonstrated in Fig. 3 b. The unetched silicon column will act as a heat sink between the $\mu \mathrm{HP}$ and the substrate preventing the temperature to reach to the desired value as shown in Fig.2 for the airpit of height less than $50 \mu \mathrm{m}$. However, for the recessed aerogel any desirable size of the pit height is achievable with a simple Manhattan mask opening. Once the pit is filled with aerogel an array of $\mu \mathrm{HPs}$ is processed on top of the recessed aerogel.

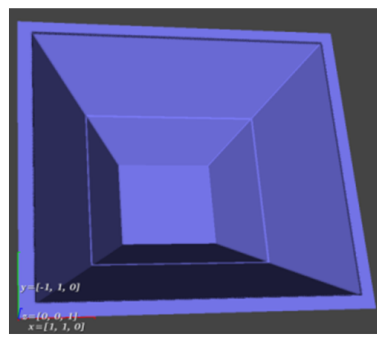

(a) Completely Etched Airpit

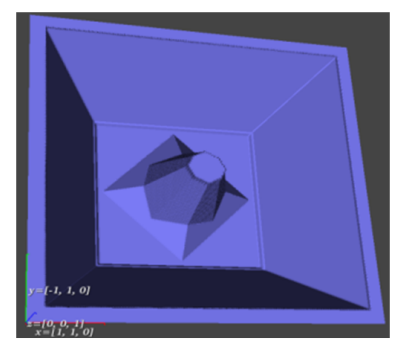

(b) Remaining Unetched Column
Fig. 3. 3D image of airpit simulated by IntelliEtch.

As shown in Fig.4, to maintain $360^{\circ} \mathrm{C}$ the power consumption by $\mu \mathrm{HP}$ array reduces exponentially as the height of the recessed aerogel increases. For the height equal and greater than $160 \mu \mathrm{m}$ the power consumed by sensor array will reach to a minimum value of $2.0 \mathrm{~mW}$.

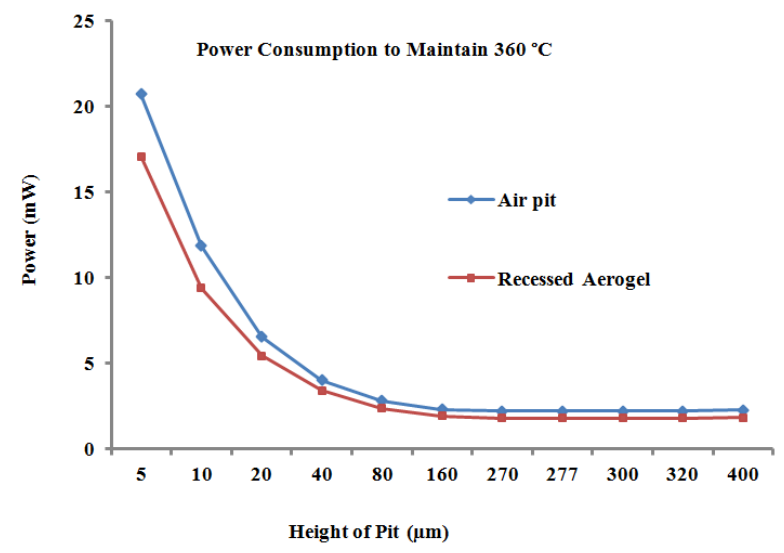

Fig. 4. Power consumption to maintain $360^{\circ} \mathrm{C}$.

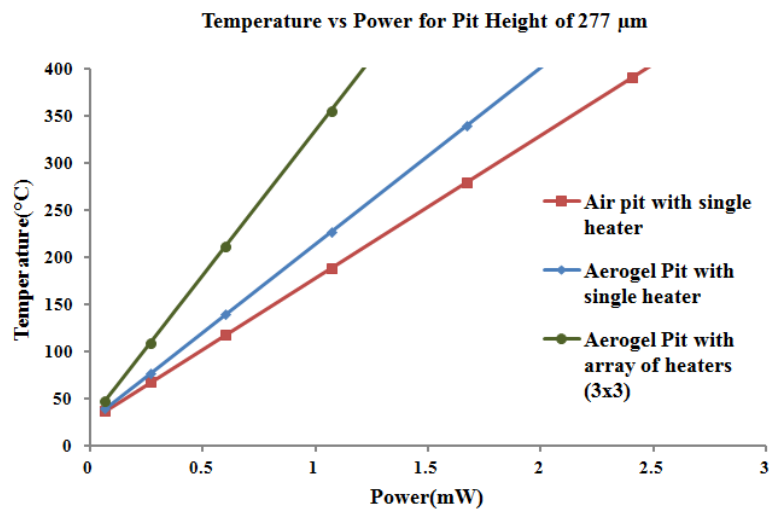

Fig. 5. Temperature vs. power for the given height of $277 \mu \mathrm{m}$

In Fig.5 temperature versus the consumed power per $\mu \mathrm{HP}$ is plotted for an air pitted, recessed aerogel, and $3 \times 3$ array 
$\left(573 \times 573 \mu \mathrm{m}^{2}\right)$ of $\mu \mathrm{HP}$ on recessed aerogel. The height of the pit is $277 \mu \mathrm{m}$. The power consumed by a $\mu \mathrm{HP}$ of an array made on the recessed aerogel gave the best result. In Fig. 2 for applied power of $2.4 \mathrm{~mW}$ per heater, the superior heat insulation of recessed aerogel is observed. However, for the air pitted $\mu \mathrm{HP}$ that the height is not sufficient to have a large $b$ for a given $a$ according to (1), the temperature would stay low because of the heat sink path through the unetched column. The two structures depicted in Fig.6 demonstrate the airpit with single heater and recessed aerogel with array of heaters.

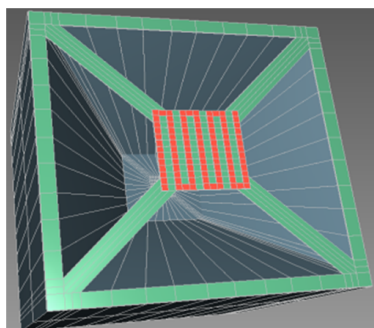

(a) Airpit

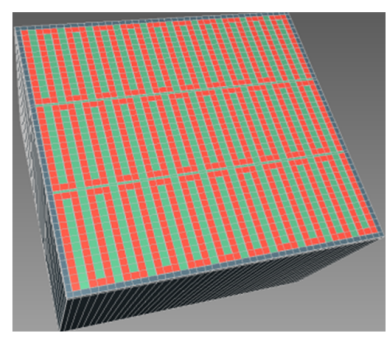

(b) Recessed aerogel
Fig. 6. 3D view of (a) air pit with single heater (b) recessed aerogel with array of heaters $(3 \times 3)$.

\section{CONCLUSION}

We have reported a remarkably efficient $\mu \mathrm{HP}$ for ultra dense gaseous sensor array that utilizes recessed aerogel in a single micromachined pit for the entire array. The recessed aerogel not only improves the efficiency of the $\mu \mathrm{HP}$ but also eliminates the problem of step coverage that can severely reduce the yield of IC-microsensors array chips in manufacturing. Moreover, by recessing aerogel the problem of multilayer processing of aerogel to obtain a thick layer is removed. Also, the recessed aerogel has the advantage of micromachining the desired cavity of any size and height prior to fabrication of the sensor arrays. In addition to the aforementioned advantages of the new structure, the recessed aerogel is shown to have extremely low power consumption as low as $3 \mu \mathrm{W} / \mu \mathrm{m}^{2}$ for $\mu \mathrm{HP}$ to maintain the temperature at $360^{\circ} \mathrm{C}$ and it can also save the area as much as ten times $\left(2.95 \times 10^{5} \mu m^{2}\right)$ compared to conventional $\mu \mathrm{HP}$ structure.

\section{REFERENCES}

[1] S. Fan, C. Kim, J. Paik, B. Dunn, P. Patterson, and M. Wu, "MEMS with Thin-Film Aerogel," in Micro Electro Mechanical Systems, 2001. MEMS 2001. The 14th IEEE International Conference on. IEEE, 2001, pp. $122-125$.

[2] H. Bargozin, L. Amirkhani, J. Moghaddas, and M. Ahadian, "Synthesis and Application of Silica Aerogel-MWCNT Nanocomposites for Adsorption of Organic Pollutants," Sci. Iran, vol. 17, pp. 122-132, 2010.

[3] J. Farmer, D. Fix, G. Mack, R. Pekala, and J. Poco, "Capacitive Deionization of $\mathrm{NaCl}$ and $\mathrm{NaNO}_{3}$ Solutions with Carbon Aerogel Electrodes," Journal of the Electrochemical Society, vol. 143, no. 1, pp. 159-169, 1996.

[4] L. Aranda, "Silica Aerogel," Potentials, IEEE, vol. 20, no. 2, pp. 12-15, 2001.
[5] S. Kim, S. Hwang, and S. Hyun, "Preparation of Carbon Aerogel Electrodes for Supercapacitor and Their Electrochemical Characteristics," Journal of Materials Science, vol. 40, no. 3, pp. 725-731, 2005.

[6] J. Arenas and M. Crocker, "Recent Trends in Porous Sound-Absorbing Materials," Sound \& Vibration, vol. 44, no. 7, pp. 12-18, 2010.

[7] K. Oh, D. Kim, and S. Kim, "Ultra-Porous Flexible PET/Aerogel Blanket for Sound Absorption and Thermal Insulation," Fibers and Polymers, vol. 10, no. 5, pp. 731-737, 2009.

[8] X. Lu, R. Caps, J. Fricke, C. Alviso, and R. Pekala, "Correlation between Structure and Thermal Conductivity of Organic Aerogels," Journal of Non-Crystalline Solids, vol. 188, no. 3, pp. 226-234, 1995.

[9] S. Prakash, C. Brinker, and A. Hurd, "Silica Aerogel Films at Ambient Pressure," Journal of Non-Crystalline Solids, vol. 190, no. 3, pp. 264275, 1995.

[10] A. Rao, G. Pajonk, S. Bhagat, and P. Barboux, "Comparative Studies on the Surface Chemical Modification of Silica Aerogels Based on Various Organosilane Compounds of the Type $\mathrm{R}_{n} \mathrm{SiX}_{4-n}$," Journal of NonCrystalline Solids, vol. 350, pp. 216-223, 2004.

[11] A. Katti, N. Shimpi, S. Roy, H. Lu, E. Fabrizio, A. Dass, L. Capadona, and N. Leventis, "Chemical, Physical, and Mechanical Characterization of Isocyanate Cross-Linked Amine-Modified Silica Aerogels," Chemistry of Materials, vol. 18, no. 2, pp. 285-296, 2006.

[12] M. Jo, J. Hong, H. Park, J. Kim, and S. Hyun, "Evaluation of $\mathrm{SiO}_{2}$ Aerogel Thin Film with Ultra Low Dielectric Constant as an Intermetal Dielectric," Microelectronic Engineering, vol. 33, no. 1, pp. 343-348, 1997.

[13] M. Madani, D. Lankireddy, and N. Tzeng, "Gaseous Sensors with Areaand Energy-Efficient Microhotplates through Silica Aaerogel for Heat Insulation," in Microelectronics (ICM), 2009 International Conference on. IEEE, 2009, pp. 402-405.

[14] I. Elmi, S. Zampolli, E. Cozzani, M. Passini, G. Cardinali, and M. Severi, "Development of Ultra Low Power Consumption Hotplates for Gas Sensing Applications," in Sensors, 2006. 5th IEEE Conference on. IEEE, 2006, pp. 243-246.

[15] I. Elmi, S. Zampolli, E. Cozzani, M. Passini, G. Pizzochero, G. Cardinali, and M. Severi, "Ultra Low Power MOX Sensors with ppb-Level VOC Detection Capabilities," in Sensors, 2007 IEEE. IEEE, 2007, pp. $170-173$.

[16] S. Fung, Z. Tang, P. Chan, J. Sin, and P. Cheung, "Thermal Analysis and Design of a Micro-Hotplate for Integrated Gas-Sensor Applications," Sensors and Actuators A: Physical, vol. 54, no. 1, pp. 482-487, 1996.

[17] D. Briand, S. Colin, A. Gangadharaiah, E. Vela, P. Dubois, L. Thiery, and N. De Rooij, "Micro-Hotplates on Polyimide for Sensors and Actuators," Sensors and Actuators A: Physical, vol. 132, no. 1, pp. 317-324, 2006.

[18] J. Laconte, D. Flandre, and J. Raskin, "Micromachined Thin-Film Sensors for SOI-CMOS Co-Integration," Micromachined Thin-Film Sensors for SOI-CMOS Co-Integration, by J. Laconte, D. Flandre, and J.-P. Raskin. 2006 XIII, 292 p. 0-387-28842-2. Berlin: Springer, 2006., vol. 1, 2006.

[19] M. Graf, D. Barrettino, H. Baltes, and A. Hierlemann, CMOS Hotplate Chemical Microsensors. Springer, 2007.

[20] M. Bauer, C. Bauer, M. Fish, R. Matthews, G. Garner, A. Litchenberger, and P. Norris, "Thin-Film Aerogel Thermal Conductivity Measurements via 3 w," Journal of Non-Crystalline Solids, vol. 357, no. 15, pp. 2960$2965,2011$.

[21] C. Wang and C. Wu, "Electrical Sensing Properties of Silica Aerogel Thin Films to Humidity," Thin Solid Films, vol. 496, no. 2, pp. 658-664, 2006.

[22] Y. Liu, L. Zhang, X. Yao, and C. Xu, "Development of Porous Silica Thick Films by a New Base-Catalyzed Sol-Gel Route," Materials Letters, vol. 49, no. 2, pp. 102-107, 2001.

[23] S. Ghandhi, VLSI Fabrication Principles: Silicon and Gallium Arsenide. John Wiley \& Sons, 2008. 